\title{
CORRECTION
}

\section{Correction to: An Instance Theory of Semantic Memory}

\section{Randall K. Jamieson ${ }^{1}$ - Johnathan E. Avery ${ }^{2} \cdot$ Brendan T. Johns $^{3} \cdot$ Michael N. Jones $^{2}$}

Published online: 29 July 2019

(C) Society for Mathematical Psychology 2019

\section{Correction to: Comput Brain Behav \\ https://doi.org/10.1007/s42113-018-0008-2}

Figure 9 in the original version of the article contained an error. The corrected Fig. 9 is presented below. Conclusions from the Instance Theory of Semantics (ITS) are preserved. However, conclusions from LSA and BEAGLE are not. Like the ITS, both LSA and BEAGLE disambiguated the meaning of a homonym when conditionalized on a disambiguating prime, whether the homonym does or does not have a dominant sense.

The online version of the original article can be found at https://doi.org/ 10.1007/s42113-018-0008-2

Michael N. Jones

jonesmn@indiana.edu

1 University of Manitoba, Winnipeg, Canada

2 Department of Psychological and Brain Sciences, Indiana University, Bloomington, USA

3 University at Buffalo, Buffalo, USA 
Fig. 9 Simulation of disambiguation with natural language. Results with ITS are shown in the top row. Results with LSA are shown in the middle row. Results with BEAGLE are shown in the bottom row. In all cases, semantic similarity is shown averaged over all 36 items from the stimulus set on the left, averaged over the 6 least dominant items in the center, and averaged over the 6 most dominant items on the right. Whiskers show the standard error of the mean computed over all comparisons in each condition

ITS

All 36

Items

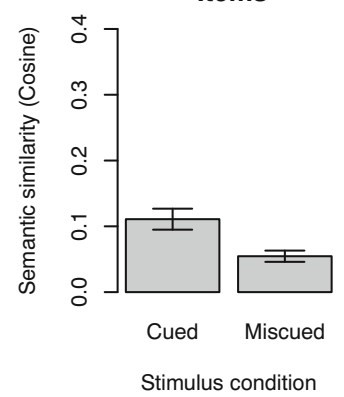

Items
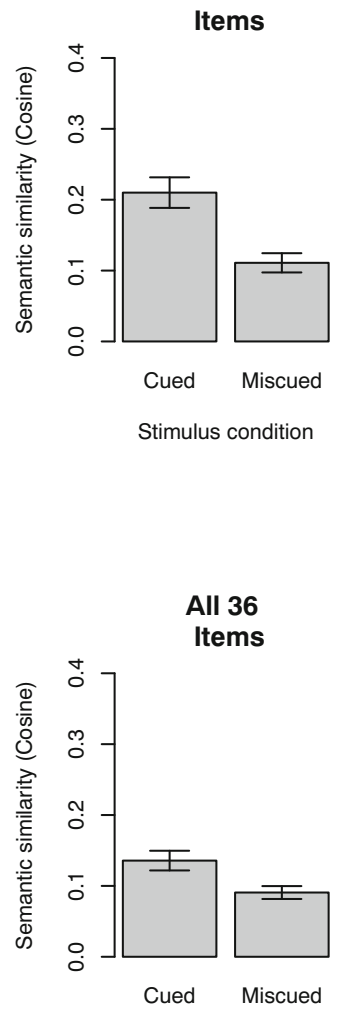

Stimulus condition
Six Least

Dominant Items

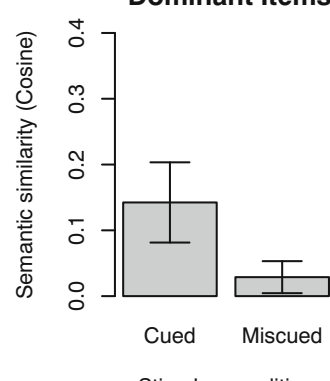

LSA

Six Least Dominant Items

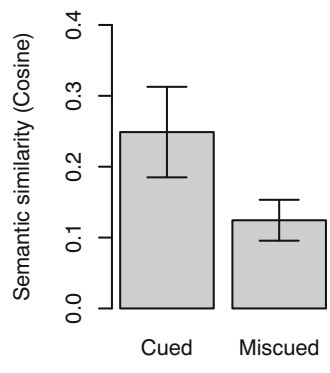

Stimulus condition

\section{BEAGLE}

Six Least Dominant Items

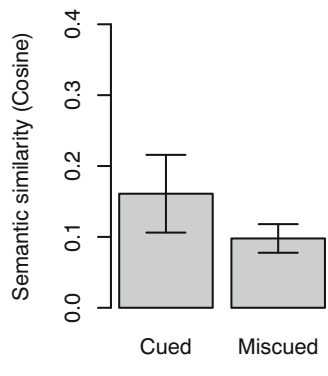

Stimulus condition
Six Most

Dominant Items

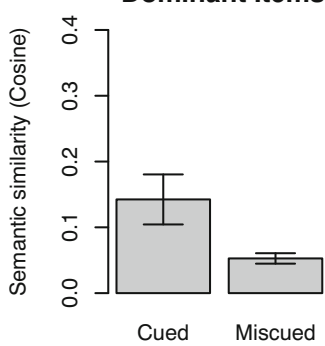

Stimulus condition

Six Most Dominant Items

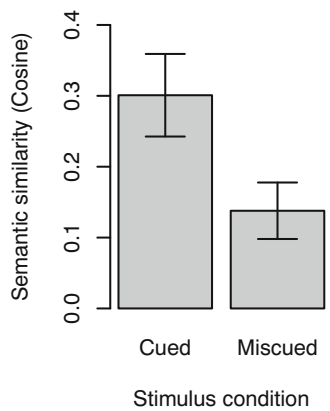

Six Most Dominant Items

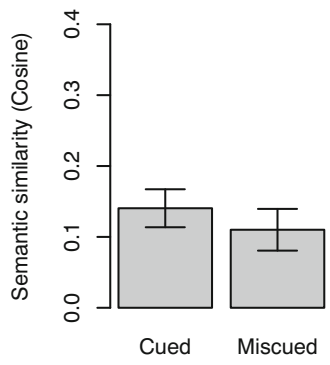

Stimulus condition

Publisher's Note Springer Nature remains neutral with regard to jurisdictional claims in published maps and institutional affiliations. 